\title{
Functional Near-Infrared Spectroscopy as a Target Navigator for rTMS Modulation in Patients with Hemiplegia: A Randomized Control Study
}

\author{
Pang-Wei Chang $\cdot$ Chia-Feng Lu $\cdot$ Shin-Tsu Chang $\cdot$ Po-Yi Tsai (D)
}

Received: September 16, 2021 / Accepted: November 1, 2021 / Published online: November 13, 2021

(c) The Author(s) 2021

\begin{abstract}
Introduction: Although repetitive transcranial magnetic stimulation (rTMS) is efficacious for motor neuromodulation in stroke survivors, high interindividual variability for responsiveness remains a concern. Target probing on the skull using a proper brain-mapping technique may help overcome this challenge. This study
\end{abstract}

Supplementary Information The online version contains supplementary material available at https:// doi.org/10.1007/s40120-021-00300-0.

P.-W. Chang · P.-Y. Tsai $(\bowtie)$

Department of Physical Medicine and

Rehabilitation, Taipei Veterans General Hospital,

Taipei, Taiwan

e-mail: pytsai@vghtpe.gov.tw

C.-F. $\mathrm{Lu}$

Department of Biomedical Imaging and

Radiological Sciences, School of Biomedical Science and Engineering, National Yang Ming Chiao Tung

University, Taipei, Taiwan

C.-F. $\mathrm{Lu}$

Institute of Biophotonics, National Yang Ming

Chiao Tung University, Taipei, Taiwan

\section{S.-T. Chang}

Department of Physical Medicine and Rehabilitation, Kaohsiung Veterans General

Hospital, Kaohsiung, Taiwan

P.-Y. Tsai

School of Medicine, National Yang Ming Chiao

Tung University, Taipei, Taiwan assessed the feasibility of functional near-infrared spectroscopy (fNIRS) as a target navigator in rTMS treatment for motor facilitation in patients with stroke.

Methods: Fifty-one patients with stroke were enrolled in this randomized controlled study. The patients were assigned to three groups: fNIRS-guided rTMS treatment (fNIRS group, $n=20$ ), motor evoked potential (MEP)-guided rTMS treatment (MEP group, $n=16$ ), and sham $(n=15)$ group. Motor assessments, including Fugl-Meyer Assessment (FMA), Wolf Motor Function Test (WMFT), and muscle strength, were conducted at baseline and after the 10-session rTMS treatment.

Results: The fNIRS-guided hotspot (fNIRS-HS) was obtained for each patient, even those for whom the MEP-guided hotspot was undetectable. Both intervention groups exhibited significant improvements in muscle strength, FMA, and WMFT scores $(P<0.001)$ compared with the sham group. The fNIRS group achieved significantly greater improvement in elbow function $(P=0.001)$ than the MEP group.

Conclusion: fNIRS can be a reliable tool for hotspot navigation for motor neuromodulation in patients with stroke. With high sensitivity to cortical oxygenation changes, this navigation system achieved a superior outcome to the traditional MEP-based method in patients with stroke. fNIRS-based systems may also facilitate the integration of machine learning, thus 
enabling precision medicine for neuromodulation.

Trial Registration: https://clinicaltrials.gov; Unique identifier: NCT02006615.

Keywords: Repetitive transcranial magnetic stimulation (rTMS); Near-infrared spectroscopy (NIRS); Stroke; Cortical excitability; Motor function

\section{Key Summary Points}

Functional near-infrared spectroscopy

(fNIRS) is a reliable tool for hotspot navigation for motor neuromodulation.

fNIRS-based hotspots can be detected in the post-stroke motor cortex with varied lesion types.

The fNIRS-based system achieved a superior outcome to the traditional motor evoked potential (MEP) method.

fNIRS may facilitate the integration of machine learning and precision medicine.

\section{INTRODUCTION}

Cerebrovascular diseases are among the ten leading causes of death in developed countries and usually result in devastating disability [1]. Although traditional neurorehabilitation is critical for post-stroke management, its general efficacy is unsatisfactory. Repetitive transcranial magnetic stimulation (rTMS), as a noninvasive brain stimulation, has become a promising tool in the treatment of post-stroke impairments under the putative mechanism of long-term potentiation (LTP) or long-term depression (LTD) [2]. rTMS delivers painless magnetic pulses to modulate cortex excitability, causing a change in the synaptic transmission followed by neuroplasticity [3]. Intermittent theta burst stimulation (iTBS), comprising compound stimulus frequencies, is stronger than the traditional paradigm in generating LTP-like or LTD-like phenomena under a shorter conditioning time $[4,5]$. iTBS over the primary motor cortex (M1) in the affected hemisphere may improve upper limb motor function in patients with chronic stroke $[3,6,7]$.

Although rTMS intervention has grade A (definitely effective) to B (probably effective) evidence for motor improvement in patients with hemiplegia following stroke [8], considerable interindividual variation exists in the response rate, which is influenced by certain factors, such as the selected target, stimulation mode, and intensity. Among them, accuracy of target localization in the affected hemisphere is a major issue. Target localization can be navigated by a frameless stereotaxic system aimed at the neuroanatomical structure instead of the functional site. However, it is best accessed by TMS navigation on the skull to probe the functional hotspot where the highest amplitude of the motor evoked potential (MEP) is evoked by the lowest intensity of magnetic output. However, the challenge with this MEP method is that the hotspot is usually undetectable, particularly in the affected hemisphere of patients with stroke $[9,10]$.

Recently, functional near-infrared spectroscopy (fNIRS) has been used to measure cortical metabolism and brain-behavior interaction, which may be a novel approach for target selection in rTMS modulation [11]. fNIRS is a unique noninvasive neuroimaging tool for functional brain mapping by monitoring brain oxygenation and hemodynamic status; it has become a promising and high-sensitivity measurement in neuroscience research [12]. Multichannel fNIRS provides insights into neurophysiological activity by detecting the signal of wavelength absorption of oxygenated hemoglobin $(\mathrm{HbO})$, deoxygenated hemoglobin (HbR), and total hemoglobin (total $\mathrm{Hb}$ ) concentration. These signals reflect the anatomical vascular supply into the functional cortex, including capillaries in the activated cortex and the downstream veins of the cortical vascular structure [13, 14]. For instance, increased hemodynamic response in $\mathrm{HbO}$ and reduction in HbR during a motor task was observed in fNIRS channels covering the primary motor area M1 and the supplementary motor area in healthy people [15-17]. In another study, increased 
HbO signal was observed shortly after the initiation of finger tapping, and this reaction was more prominent in the channel located in M1 [18]. These previous findings support the high specificity of fNIRS in capturing the center of activation during cortical mapping.

Although fNIRS mapping has a lower spatial resolution than functional magnetic resonance imaging (fMRI) in differentiating active brain regions, it is unknown whether fNIRS can be used as a navigator to locate the optimal region for rTMS treatment. While high-density fNIRS can achieve millimeter-scale resolution, the regular-density fNIRS (source detector distance: $\sim 3 \mathrm{~cm}$ ) can reach a spatial resolution of up to $1 \mathrm{~cm}[19,20]$. Cao et al. used the 24-channel fNIRS of diffuse optical tomography as the spatial prior to electroencephalography (EEG) and demonstrated high spatiotemporal resolution in detecting neuronal sources using a combined EEG-fNIRS system [20]. Recently, a set of fNIRS probes was designed to aid transcranial direct current stimulation in the feedback analysis of electric field distribution during cortical modulation [21]. Although fNIRS is sufficiently sensitive to detect cortical $\mathrm{Hb}$ change related to active central or electrophysiological drive, little is known about the feasibility of fNIRS as a surrogate for MEP to navigate targets for rTMS treatment in patients with hemiplegia.

Based on the rationale that fNIRS has high reliability in cortical activation measurement and high sensitivity to cortical excitability change, we hypothesized that for target determination, fNIRS incorporated into rTMS would be ideal for motor facilitation following stroke. In this randomized controlled trial (RCT), we divided hemiplegia patients into different groups to elucidate the possible differential effects of motor facilitation exerted by excitatory rTMS guided by fNIRS or the traditional MEP method.

\section{METHODS}

\section{Participants}

Individuals with stroke were recruited from a tertiary medical center from November 2018 to April 2020. Data on age, sex, stroke type, lesion side, and duration of hemiparesis were obtained from interviews and medical charts. To be included in the study, participants with stroke had to satisfy the following criteria: (1) experienced a single stroke, with 2 months to 2 years since onset; (2) age between 30 and 80 years; and (3) hemiparesis with upper limb weakness and functional impairment. Participants were excluded if they had (1) a contraindication for rTMS treatment: presence of cardiac pacemakers or implantable cardioverter defibrillators, metal or magnetic objects in the brain, ear implants, aneurysm clips or coils, metal plates in the head/brain, and medication pumps for people; (2) a history of epilepsy; (3) visual defect or achromatopsia; or (4) the presence of other neurological diseases such as Parkinson's disease or dementia. All participants provided written informed consent. The study protocol was approved by the Institutional Review Board of Taipei Veterans General Hospital (201307011A). All procedures performed in this study involving human participants were in accordance with the ethical standards of the national research committee and with the 1964 Helsinki Declaration and its later amendments.

The $G^{*}$ Power program (v3.1.9.2; Franz Faul, University of Kiel, Kiel, Germany) was used to calculate the necessary sample size [22]. On the basis of a previous study using rTMS-iTBS on the ipsilesional M1 for upper limb motor improvement in patients with chronic stroke [23], an effect size of 0.53 was selected. In the one-way analysis of variance (ANOVA) test, an $\alpha$-error of 0.05 and a $\beta$ of 0.20 (power level of 0.80 ) were used, indicating a minimum of 39 patients. Assuming a dropout rate of $15 \%$ and the fact that we had three groups (one sham and two intervention), at least 15 patients per group were required.

\section{Study Design}

This was a prospective, double-blind, shamcontrolled study. The randomization order was computer-generated and concealed in sequentially numbered opaque envelopes by an independent statistician. The patients were randomly assigned to one of the following three 
groups in a 1:1:1 allocation ratio: those receiving rTMS-iTBS stimulation guided by fNIRS (fNIRS group), those receiving rTMS-iTBS stimulation guided by TMS-MEP (MEP group), or those receiving sham stimulation (sham group) guided by TMS-MEP. All groups underwent 10 daily sessions, 5 days per week, for 2 weeks. In the fNIRS and MEP groups, iTBS was applied on the ipsilesional fNIRS-guided hotspot (fNIRSHS) and ipsilesional MEP-guided hotspot (MEPHS), respectively. In cases where the hotspot was not obtainable in the affected hemisphere, the mirror site of contralesional M1 was used, with iTBS as the stimulation paradigm in the MEP group and the sham group. Both positions of fNIRS-HS and MEP-HS were recorded in the international 10-5 system, and their distances were calculated. Brunnstrom stage, Medical Research Council (MRC) scale, the Fugl-Meyer Assessment (FMA) score, the Wolf Motor Function Test (WMFT) score, and the grasping strength in the paretic upper limb were measured before and after the intervention in all patients (Fig. 1). Clinical assessment was conducted by an experienced physician blinded to patients' group allocation, and rTMS was performed by another investigator who was not involved in clinical assessment, patient followup, or data analysis.

\section{fNIRS Imaging System}

A multichannel wearable fNIRS imaging system (NIRSport, NIRx Medical Technologies, LLC, Glen Head, NY, USA) was used to simultaneously acquire dual-wavelength (760 and $850 \mathrm{~nm}$ ) signals. The fNIRS optodes, including eight LED light sources and eight detectors in each hemisphere, were attached to participants' heads to monitor the hemodynamics of the bilateral motor cortex (Fig. 2).

The calculation of brain hemodynamic signals is provided as a supplementary document. In summary, the preprocessed signals were converted into concentration changes in oxygenated hemoglobin, $\Delta[\mathrm{HbO}]$, and deoxygenated hemoglobin, $\Delta[\mathrm{HbR}]$, using the modified Beer-Lambert law for each source-detector channel study [24].
The fNIRS head cap was specifically designed in compatibility with the international 10-5 system, which defines standard surface positions for a human head, with approximately $3.0 \mathrm{~cm}$ between any two adjacent positions. In the source and detector arrangements, 23 effective source-detector channels were generated for monitoring local blood oxygenation, with a sampling rate of $6.25 \mathrm{~Hz}$. During signal acquisition, the time points for the beginning of each task condition were recorded.

To estimate the signal-to-noise quality of a data channel, the relative coefficient of variation (CV, in \%) was calculated for the raw signals at $760 \mathrm{~nm}$ and $850 \mathrm{~nm}$, which is a routine procedure for fNIRS measurement [23]. Data rejection based on two types of $\mathrm{CV}, \mathrm{CV}_{\text {chan }}$, and $\mathrm{CV}_{\text {trial, }}$ was used to reduce physical artifacts, such as motion-induced instability and blood pressure-induced hemodynamics [25].

$$
\mathrm{CV}=\sigma / \mu \times 100 \%,
$$

where $\mu$ is the mean value and $\sigma$ is the standard deviation of the signal. $\mathrm{CV}_{\text {chan }}$ was calculated over the entire duration of the experiment (approximately $13 \mathrm{~min}$ ) for each channel, and measurement channels with $\mathrm{CV}_{\text {chan }}>15 \%$ were rejected. The $\mathrm{CV}_{\text {trial }}$ was then obtained for 60-s intervals of the individual trial block, and only trials for each remaining channel $\left(\mathrm{CV}_{\text {chan }}<15 \%\right)$ with $\mathrm{CV}_{\text {trial }}<10 \%$ in both wavelengths were retained for subsequent analyses.

The remaining fNIRS signals were bandpassfiltered (low-cutoff frequency $0.005 \mathrm{~Hz}$ and high-cutoff frequency $0.03 \mathrm{~Hz}$ ) to eliminate the effects of heartbeat, respiration, and low-frequency signal drifts for each wavelength [25]. Wavelet filtering was subsequently used to correct for the motion artifacts in each channel. The measured signal is assumed to be a linear combination of the physiological signal of interest (hemodynamics) and motion artifacts. Because the hemodynamic response is much slower than motion artifacts (such as a spike artifact), the wavelet coefficients for the evoked response are anticipated to be a Gaussian probability distribution [26, 27]. Motion artifacts usually cause a sudden change in signal 


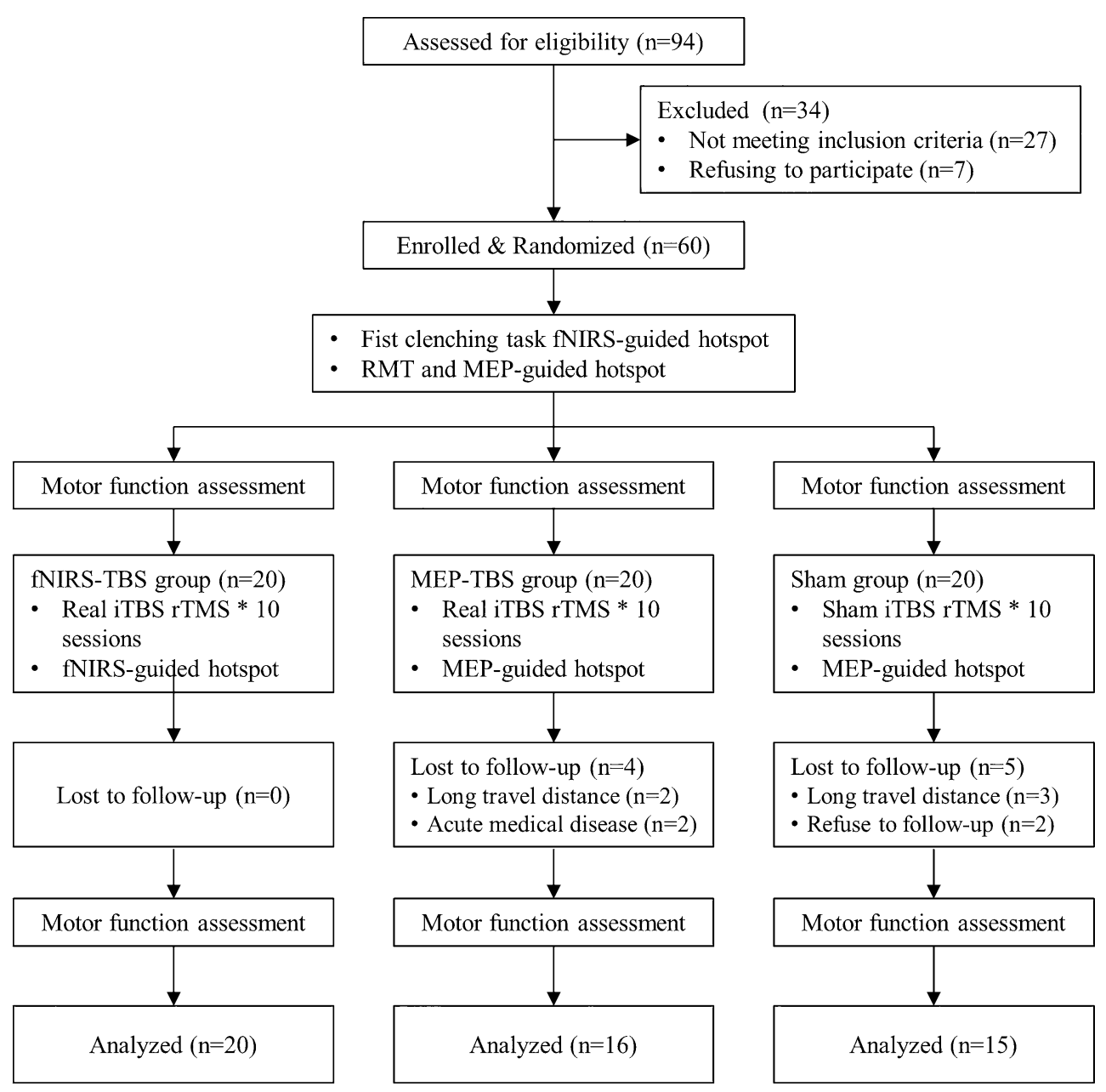

Fig. 1 CONSORT flowchart

amplitude with a large coefficient in the wavelet domain. Accordingly, the wavelet coefficients of hemodynamics are around zero, with smaller variance compared to the coefficients of motion artifacts which can be identified as outliers. The outlying coefficients (larger than a predefined threshold) were therefore eliminated before signal reconstruction using the inverse discrete wavelet to correct the corresponding motion artifacts. The selected threshold value for artifact removal is based on the level of artifact contamination. The removal threshold of the wavelet coefficient, $\alpha$, was set to 0.1 in this study based on a previous study [28]. The preprocessed signals were converted to concentration changes in oxygenated hemoglobin, $\Delta[\mathrm{HbO}]$, and deoxygenated hemoglobin, $\Delta[\mathrm{HbR}]$, using the modified Beer-Lambert law for each sourcedetector channel study [21]. Correlation-based signal improvement (CBSI) was then employed to improve the signal quality based on the findings that brain activation involves $\Delta$ [HbO] increases and $\Delta[\mathrm{HbR}]$ decreases at the activated cortical regions [29]. The relative changes in $[\mathrm{HbO}]$ and $[\mathrm{HbR}]$ concentrations (i.e., $\Delta[\mathrm{HbO}]$ and $\Delta[\mathrm{HbR}])$ were calculated based on a 5-s baseline and collected during the task of hand grasp. Participants were asked to keep clenching and relaxing their fists alternately for $20 \mathrm{~s}$ and then rest for $20 \mathrm{~s}$, repeatedly for five cycles while fNIRS was recorded. The sequence of right or left hand examined was in random order for each patient. Patients with a completely paretic hand (i.e., unable to make a fist) were taught to 


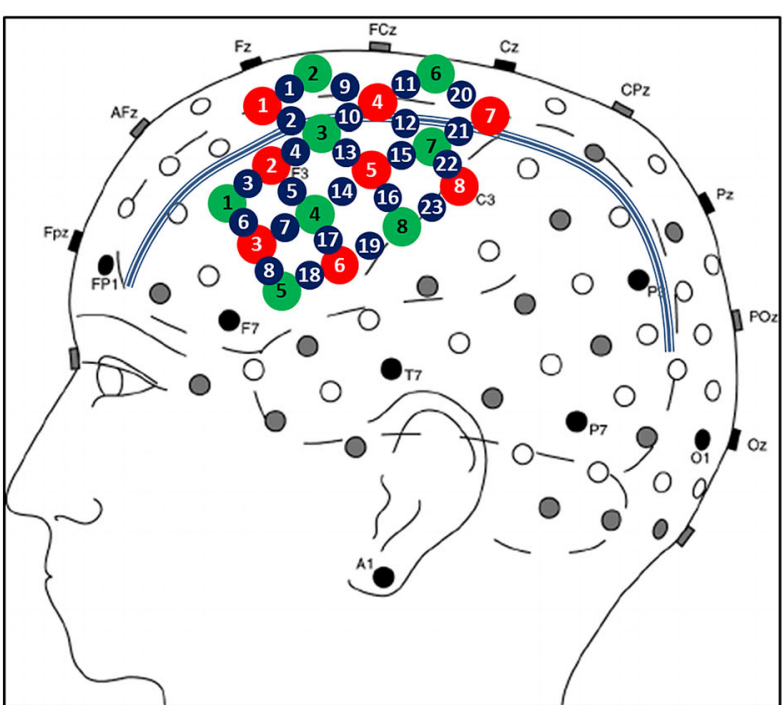

Fig. 2 Arrangement of fNIRS probes covering the bilateral premotor cortex, primary motor cortex (M1), and supplemental motor area (SMA). $\Delta[\mathrm{HbO}]$ at S4D2

perform an imagery task (i.e., imagine they were holding a cup to drink or grooming their hair). The fNIRS signal preprocessing, including the motion artifact correction, bandpass filtering, and conversion of $[\mathrm{HbO}]$ and $[\mathrm{HbR}]$, were processed using the HOMER2 package [30]. The calculation of signal CVs and correlation analyses were performed using homemade scripts developed on MATLAB (MathWorks, Natick, MA, USA). The channel that yielded the most vigorous [HbO] response of the block average was chosen as the treatment target for the NIRS group. In the pilot study of our previous trial, the $\Delta[\mathrm{HbR}]$ did not show a consistent response to the grasp task; therefore, we used $\Delta[\mathrm{HbO}]$ as a biomarker of fNIRS for the following analyses in this study. The use of $\Delta[\mathrm{HbO}]$ as a biomarker was based on studies whereby a significant change in [HbO] was noted in channels covering the primary motor cortex during the fingertapping task in healthy subjects [31].

\section{Measurements of Motor Function}

We adopted the Brunnstrom stages, MRC scale, FMA, WMFT, and grip strength as the outcome evaluations for motor performance. WMFT served as the primary outcome measurement.

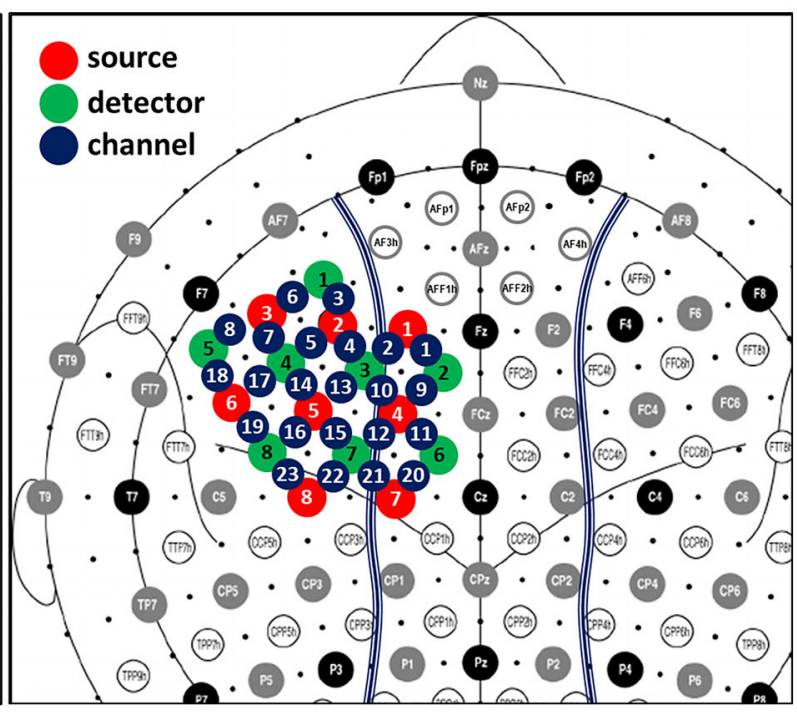

$\mathrm{CH}$ 9, S5D3 CH13, S5D8 CH16, and S8D7 CH 22 yielded significant correlation with RMT in the contralesional hemisphere

All the patients were assessed before the rTMS intervention and the next day after completing the treatment.

The Brunnstrom stages, known as the approach for stroke improvement, classify motor progress into a series of six distinct stages. We used the MRC scale to determine muscle strength ranging from grades 0 (no visible contraction) to 5 (normal) of six muscle groups of the shoulder flexor, elbow extensor, elbow flexor, wrist extensor, wrist flexor, and hand grasp muscles. The FMA is a well-known assessment tool for measuring post-stroke physical impairments related to functional recovery and assessing the ability to isolate movements at each joint and the influence of unwanted synergies on movement. The FMA has seven domains related to the upper extremity (upper extremity, wrist, hand, coordination/speed, sensation, passive range of motion, and joint pain) and includes 63 items (score range, 0-126) [32]. The WMFT evaluates upper extremity motor function through the use of timed and functional tasks. The widely used version of the WMFT consists of 16 items, namely forearm to table (side), forearm to box (side), extended elbow (side), extended elbow (to the side) with $1 \mathrm{lb}$ weight, hand to 
table (front), hand to box (front), weight to box, reach and retrieve, lift a can, lift a pencil, pick up paper clip, stack checkers, flip cards, turn a key in a lock, fold towel, and lift a basket. The first six items involve timed functional tasks, followed by one to measure strength, and the remaining nine items assess movement quality when completing various tasks [33]. The items are rated on a six-point scale [34]. The grip strength was tested using a digital hand dynamometer (Jamar Plus+, Patterson Medical, Cedarburg, WI, USA), which was set in line with the patient's forearm at the thigh level and squeezed as hard as possible.

A Dantec Keypoint electromyograph (Dantec, Skovlunde, Denmark) was connected to the stimulator to record MEP signals [35]. The amplified (100 $\mu \mathrm{V}-1 \mathrm{mV} /$ div) and bandpass-filtered $(20-2000 \mathrm{~Hz})$ signals were digitized at a sampling rate of $20 \mathrm{kHz}$. Each patient sat in a comfortable reclining armchair, with both hands relaxed and keeping their eyes open. We recorded MEPs bilaterally from the first dorsal interosseous hand muscles using surface $\mathrm{Ag} /$ $\mathrm{AgCl}$ electrodes. A grid of 49 positions, spaced $1 \mathrm{~cm}$ on the interaural and sagittal lines, was tested on each motor cortex. A hotspot was defined as the position where the highest amplitude could be evoked with the lowest stimulation intensity. We defined the resting motor threshold (RMT) for MEP as the lowest intensity at which MEPs $\geq 50 \mu \mathrm{V}$ amplitude could be elicited in half of 10 single-pulse consecutive TMS stimuli at the hotspot.

\section{Repetitive Transcranial Magnetic Stimulation Protocol}

We performed TMS with the Magstim Rapid $^{2}$ stimulator (Magstim Company Ltd, Whitland, Dyfed, UK) using a 70-mm figure-ofeight coil. iTBS consisted of bursts containing three $50-\mathrm{Hz}$ pulses every $200 \mathrm{~ms}$ for $2 \mathrm{~s}$ and repeated every $10 \mathrm{~s}$ for 20 cycles and 600 pulses [4]. The intensity of iTBS was set at $80 \%$ of RMT over the ispilesional target area in both the MEP and fNIRS groups. The stimulation protocol for sham control was identical to the experimental group, but a placebo coil (Magstim) was used for the sham stimulation; this delivered $<5 \%$ of the magnetic output with audible click-on discharge. Because none of the patients had ever undergone rTMS, they could not identify whether the stimulation was real or sham. Each patient received iTBS intervention for 10 daily sessions ( 5 days per week for 2 weeks).

\section{Statistical Analysis}

Statistical analysis was performed using SPSS 22.0 software (IBM Corp., Armonk, NY, USA). Demographic data including sex, involved laterality, type of stroke, and location of lesion were compared by group using a chi-square test. However, age, duration from stroke onset to the experimental day, and baseline upper limb motor assessment were compared using oneway ANOVA. For intragroup comparison, we used a paired $t$ test to evaluate the group-wise motor improvement. To compare the change scores of motor performance among the three groups, we used one-way ANOVA and a post hoc analysis with Bonferroni's correction. To obtain the relationship between fNIRS expression and neurophysiological and clinical behavior, we used Spearman's correlation to analyze the values of $\Delta[\mathrm{HbO}]$ and RMT. To clarify the relationship between the distance of the fNIRS-HS from the mean MEP-HS and the motor improvement, we correlated the distance and change scores of motor performance in the fNIRS group using Spearman's correlation analyses. The level of significance was set at $P<0.05$. All data are expressed as mean $\pm s$ tandard deviation.

\section{RESULTS}

\section{Demographic and Clinical Characteristics}

We recruited 94 patients with stroke and upper limb weakness. Of these, 27 did not meet the inclusion criteria, and seven declined participation. Finally, 60 patients were included in the study from November 2018 to April 2020 and were divided into the fNIRS (experimental group), the MEP (positive control), and the 
Table 1 Demographic data and motor assessment scores at baseline for each group in mean (SD)

\begin{tabular}{|c|c|c|c|c|c|}
\hline Characteristics & All $(n=51)$ & fNIRS $(n=20)$ & $\operatorname{MEP}(n=16)$ & Sham $(n=15)$ & $P$ value \\
\hline Male sex, $n(\%)$ & $34(67)$ & $12(60)$ & $10(63)$ & $12(80)$ & 0.422 \\
\hline Age (years) & $58.4(11.8)$ & $58.9(12.6)$ & $54.9(11.3)$ & $61.4(10.9)$ & 0.303 \\
\hline Period post-stroke (months) & $12.6(8.5)$ & $12.2(7.9)$ & $14.3(9.0)$ & $11.2(9.0)$ & 0.587 \\
\hline Lesion site (right/left) & $31 / 20$ & $12 / 8$ & $12 / 4$ & $7 / 8$ & 0.270 \\
\hline Stroke type & & & & & 0.342 \\
\hline Infarction, $n(\%)$ & $32(63)$ & $14(70)$ & $9(56)$ & $9(60)$ & \\
\hline Hemorrhage, $n(\%)$ & $14(27)$ & $3(15)$ & $5(31)$ & $6(40)$ & \\
\hline Mixed, $n(\%)$ & $5(10)$ & $3(15)$ & $2(13)$ & $0(0)$ & \\
\hline Location & & & & & 0.744 \\
\hline Cortical, $n(\%)$ & $15(29)$ & $6(30)$ & $5(31)$ & $4(27)$ & \\
\hline Subcortical, $n(\%)$ & $25(49)$ & $8(40)$ & $8(50)$ & $9(60)$ & \\
\hline Mixed, $n(\%)$ & $11(22)$ & $6(30)$ & $3(19)$ & $2(13)$ & \\
\hline Brunnstrom, proximal UE (1-6) & $3.3(1.4)$ & $3.4(1.5)$ & $3.4(1.3)$ & $3.1(1.4)$ & 0.835 \\
\hline Brunnstrom, distal UE (1-6) & $3.1(1.6)$ & $3.2(1.5)$ & $3.3(1.5)$ & $2.9(1.5)$ & 0.844 \\
\hline \multicolumn{6}{|l|}{ MRC (0-5) } \\
\hline Shoulder flexion & $2.9(1.3)$ & $3.1(1.2)$ & $2.9(1.5)$ & $2.7(1.4)$ & 0.646 \\
\hline Elbow extension & $2.5(1.4)$ & $2.4(1.2)$ & $2.8(1.5)$ & $2.4(1.5)$ & 0.706 \\
\hline Elbow flexion & $3.1(1.3)$ & $3.3(1.1)$ & $3.3(1.3)$ & $2.7(1.4)$ & 0.277 \\
\hline Wrist extension & $1.4(1.2)$ & $1.2(1.0)$ & $1.5(1.3)$ & $1.5(1.3)$ & 0.593 \\
\hline Wrist flexion & $1.4(1.3)$ & $1.2(1.1)$ & $1.6(1.4)$ & $1.5(1.3)$ & 0.593 \\
\hline Hand grasping & $2.0(1.3)$ & $1.8(1.1)$ & $2.4(1.3)$ & $1.9(1.4)$ & 0.299 \\
\hline FMA $(0-126)$ & $84.7(19.4)$ & $82.5(18.8)$ & $86.5(18.7)$ & $85.7(21.8)$ & 0.812 \\
\hline WMFT (0-75) & $26.7(21.9)$ & $24.5(21.0)$ & $27.6(20.6)$ & $28.7(25.3)$ & 0.844 \\
\hline Grip strength (kg) & $4.9(4.4)$ & $5.4(4.2)$ & $5.3(5.2)$ & $3.9(3.8)$ & 0.576 \\
\hline
\end{tabular}

Analysis method with ANOVA and chi-square test

$U E$ upper extremity, MRC Medical Research Council scale, FMA Fugl-Meyer Assessment, WMFT Wolf Motor Function Test

sham groups (negative control), with 20 patients each. No adverse events were reported during the treatment. No significant betweengroup differences were noted in demographics or baseline functional scores, including the period post-stroke, type of stroke, and location of lesion (Table 1).

\section{fNIRS-Guided Hotspot (fNIRS-HS)}

Figure 3 illustrates the motion correction performance of fNIRS signals in one of the patients as an example.

Figure 4 presents a comparison of paired MEP-HS and fNIRS-HS in the contralesional 
hemisphere. The mean group locations of MEPHS and fNIRS-HS were at $(X: 35.4, Y: 22.7 \mathrm{~mm})$ and $(X: 32.0, Y: 19.8 \mathrm{~mm})$ from $\mathrm{Cz}$, respectively. The mean distance between MEP-HS and fNIRSHS was $17.7 \mathrm{~mm}$ (SD: 7.5). The fNIRS-HS demonstrated a mean medial displacement of $2.5 \mathrm{~mm}$ (SD: 12.2) and a mean posterior displacement of $3.0 \mathrm{~mm}$ (SD: 15.4) compared with the MEP-HS. Notably, in cases where the hotspot could not be obtained through MEP probing $(n=24$ of 51$)$, we could still locate the fNIRS-HS, defined as the channel with the most vigorous change in $[\mathrm{HbO}]$ signal. The correlation analyses indicated that with a shorter distance between the fNIRS-HS and mean MEP-HS, the fNIRS-guided method achieved greater motor improvement in elbow extension strength as measured with WMFT $(r=-0.508$, $P=0.026)$.

\section{Correlation Analyses for fNIRS Signals and RMT}

To determine the reliability of fNIRS, we correlated the hemodynamic responses of $\Delta[\mathrm{HbO}]$ in each channel and cortical excitability assessed using RMT in the contralesional hemisphere. Significant correlations were noted at the channels covering the M1 and S1 areas: S4D2 (CH9, $r=-0.663, P=0.037)$, S5D3 (CH13, $r=-0.672, P=0.023)$, S5D8 (CH16, $r=-0.720$, $P=0.012)$, and S8D7 (CH22, S1 area, $r=-0.782, P=0.013$ ) (Fig. 2, Table 2). Because the MEP recorded in the paretic hand was only obtainable in four patients, the RMT was present in a very small number of cases, thus precluding the correlation for ipsilesional analysis.

\section{Group-Wise Improvements}

The fNIRS group exhibited significant improvement in proximal Brunnstrom stage following rTMS intervention $(P=0.042)$, whereas no significant change was observed in the MEP or sham groups (Table 3). For muscle strength, significant improvements were noted in proximal and distal muscles in both intervention groups: shoulder flexion $(P<0.001)$, elbow extension $(P=0.002)$, elbow flexion
$(P<0.001)$, wrist extension $(P=0.021)$, wrist flexion $(P=0.021)$, and grasping $(P<0.001)]$ in the fNIRS group, and shoulder flexion $(P=0.001)$, elbow flexion $(P=0.003)$, and grasping $(P<0.001)$ strength in the MEP group. No significant changes in muscle strength were observed in the sham group. For the FMA total score, both intervention groups exhibited significant improvement (fNIRS group: $P<0.001$; MEP group: $P=0.009$ ) compared with their baseline levels. For WMFT, we observed that total scores increased significantly in both intervention groups (both $P<0.001$ ). For subtests of WMFT, the fNIRS group exhibited significant improvements in each sub-test for gross motor assessment $(P<0.001-0.01)$ compared with baseline levels. The MEP group exhibited significantly increased sub-scores in the forearm $(P=0.002)$ and reach and retrieve $(P=0.001)$. The sham group also improved in the forearm to table sub-test $(P=0.041)$. In addition, both the fNIRS $(P=0.001)$ and MEP $(P=0.006)$ groups had significant enhancement of grip strength (Table 3 ).

\section{Intergroup Comparisons}

One-way ANOVA and post hoc analyses comparing the fNIRS and MEP groups revealed that the fNIRS group exhibited superior results in elbow extension assessed with weight resistance $[F(2,48)=6.601, P=0.003$, post hoc: $P=0.010]$ or without resistance $[F(2,48)=12.471$, $P<0.001$, post hoc: $P=0.001$ ] in WMFT (Table 3, Fig. 5). Post hoc fNIRS and sham group comparison revealed significant differences in the following: FMA total score $[F(2,48)=5.467$, $P=0.033]$, WMFT total score $[F(2,48)=11.973$, $P<0.001]$, sub-tests of weight of grasping $[F(2,48)=4.461, \quad P=0.016]$, and strength of shoulder flexor $[F(2,48)=6.729, \quad P=0.002]$, elbow extensor $[F(2,48)=4.316, \quad P=0.024]$, elbow flexor $[F(2,48)=3.431, P=0.036]$, and grasp muscles $[F(2,48)=8.708, P<0.001]$. For MEP and sham group comparison, significant differences were observed in the following: FMA total score $[F(2,48)=5.467, P=0.010]$, WMFT total score $[F(2,48)=11.973, P=0.010]$, upper extremity sub-score $[F(2,48)=4.183, P=0.029]$, 
S4-D7
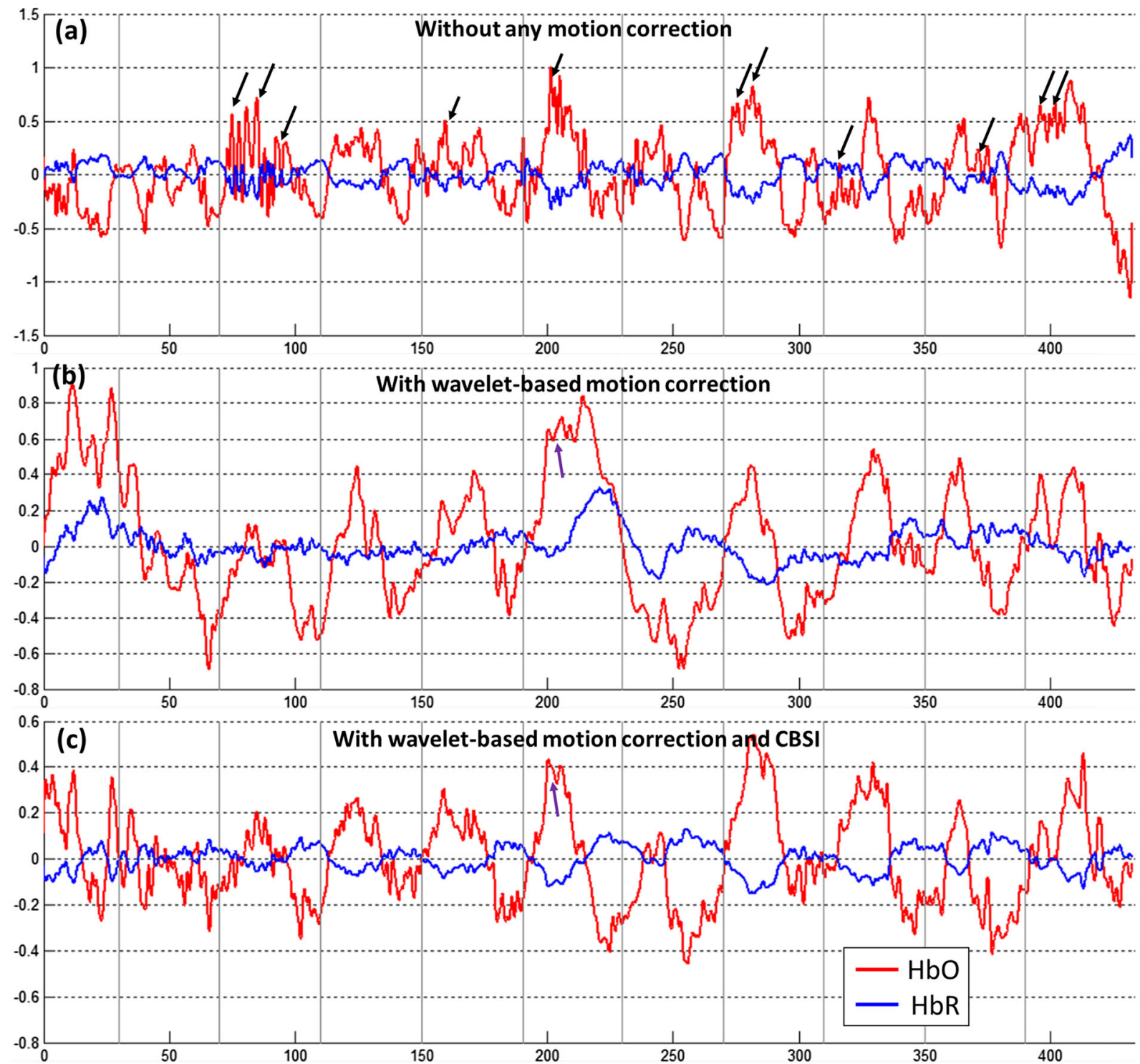

Fig. 3 a Shows that in the reconstructed $[\mathrm{HbO}]$ signals without any motion correction, contamination of spike noise is observed (black arrows). In $\mathbf{b}$, the spikes are effectively eliminated using the wavelet-based method. However, an overestimation of $[\mathrm{HbO}]$ response (a relatively stronger amplitude compared to other blocks) was identified (the purple arrow). In c, the additional CBSI processing, with the consideration of both [HbO] and $[\mathrm{HbR}]$ signal profiles, further reduces this overestimation of $[\mathrm{HbO}]$ response and restores the negative correlation between $[\mathrm{HbO}]$ and $[\mathrm{HbR}]$ changes

proximal or distal upper limb function after iTBS modulation, with no intergroup differences except in elbow function, which had a superior outcome in the fNIRS group. 


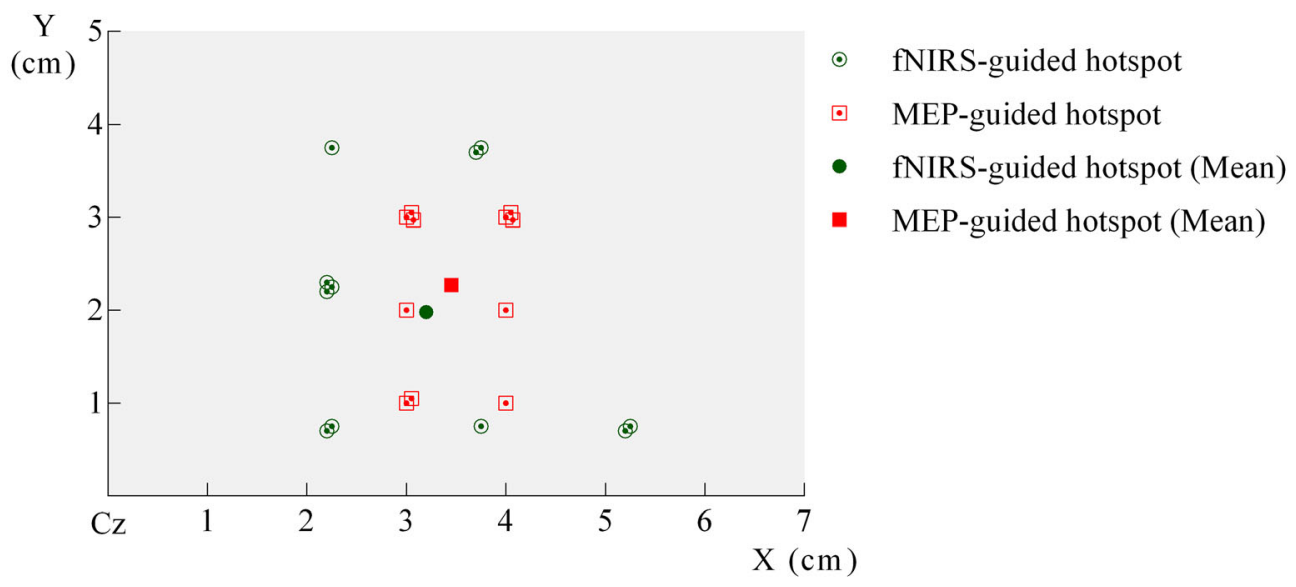

Fig. 4 Location of MEP-guided and ANIRS-guided hotspot related to $\mathrm{Cz}$ position on the skull surface. The same numbers indicate paired fNIRS-HS and MEP-HS. Green color: fNIRS-HS, red color: MEP-HS, solid circle or solid square: mean group location

Table 2 Correlation analyses for RMT and $\Delta[\mathrm{HbO}]$ signals in channels of fNIRS

\begin{tabular}{|c|c|c|c|c|c|c|c|c|c|c|c|c|}
\hline \multicolumn{13}{|c|}{ Spearman's correlation between RMT and $\Delta[\mathrm{HbO}]$ signal of NIRS } \\
\hline \multirow[t]{2}{*}{ Channel } & S1D2 & S1D3 & S2D1 & S2D3 & S2D4 & S3D1 & S3D4 & S3D5 & $\mathrm{S} 4 \mathrm{D} 2^{*}$ & S4D3 & S4D6 & S4D7 \\
\hline & $\mathrm{CH} 1$ & $\mathrm{CH} 2$ & $\mathrm{CH} 3$ & $\mathrm{CH} 4$ & $\mathrm{CH} 5$ & $\mathrm{CH} 6$ & $\mathrm{CH} 7$ & $\mathrm{CH} 8$ & $\mathrm{CH} 9$ & $\mathrm{CH} 10$ & $\mathrm{CH} 11$ & $\mathrm{CH} 12$ \\
\hline Spearman's $r$ & -0.576 & -0.527 & -0.025 & -0.310 & -0.222 & 0.193 & -0.201 & -0.181 & -0.663 & -0.457 & -0.393 & -0.363 \\
\hline$P$ value & 0.064 & 0.095 & 0.943 & 0.354 & 0.511 & 0.570 & 0.553 & 0.595 & 0.037 & 0.184 & 0.232 & 0.273 \\
\hline \multirow[t]{2}{*}{ Channel } & S5D3* & S5D4 & S5D7 & S5D8* & S6D4 & S6D5 & S6D8 & S7D6 & S7D7 & $\mathrm{S} 8 \mathrm{D} 7^{*}$ & S8D8 & \\
\hline & $\mathrm{CH} 13$ & $\mathrm{CH} 14$ & $\mathrm{CH} 15$ & $\mathrm{CH} 16$ & $\mathrm{CH} 17$ & $\mathrm{CH} 18$ & $\mathrm{CH} 19$ & $\mathrm{CH} 20$ & $\mathrm{CH} 21$ & $\mathrm{CH} 22$ & $\mathrm{CH} 23$ & \\
\hline Spearman's $r$ & -0.672 & -0.377 & -0.601 & -0.720 & -0.338 & 0.179 & 0.009 & -0.335 & -0.040 & -0.782 & -0.632 & \\
\hline$P$ value & 0.023 & 0.253 & 0.087 & 0.012 & 0.310 & 0.620 & 0.978 & 0.313 & 0.906 & 0.013 & 0.055 & \\
\hline
\end{tabular}

\section{DISCUSSION}

This double-blind, randomized controlled study investigated the effectiveness of the fNIRS-aided navigation system in rTMS treatment for motor facilitation in stroke-related hemiplegia. We demonstrated that fNIRS can reliably assess cortical hemodynamic activity and can be a surrogate for conventional MEP-defined targets for rTMS treatment. In the bilateral motor cortex where the MEP-HS was unobtainable, fNIRS yielded excellent sensitivity and topographical resolution in hotspot localization, which compensates for the inadequacy of the conventional method. Furthermore, we demonstrated the superior therapeutic effect achieved by the fNIRS method reflected in elbow functional performance after rTMS intervention compared with the MEP-guided method. With fNIRS assistance, patients demonstrated more favorable outcomes in elbow, wrist, and hand function compared with the sham group. We first explored the promising effect of the fNIRS navigation system for rTMS protocol refinement to optimize the neurostimulation effect in patients with hemiplegia.

Suppressive low-frequency $(\leq 1 \mathrm{~Hz})$ rTMS of contralesional M1 in the post-acute stage of stroke reached level A evidence (definitely effective) for hand motor recovery, whereas excitatory high-frequency rTMS $(>5 \mathrm{~Hz})$ of ipsilesional M1 has reached level $\mathrm{B}$ evidence (probably effective) [8]. Because the phenomenon of interhemispheric activity 
Table 3 Mean group data for motor assessments and group-wise and intergroup comparisons

\begin{tabular}{|c|c|c|c|c|c|c|}
\hline & \multicolumn{2}{|l|}{ fNIRS $(n=20)$} & \multicolumn{2}{|l|}{$\operatorname{MEP}(n=16)$} & \multicolumn{2}{|l|}{ Sham $(n=15)$} \\
\hline & Pre-rTMS & Post-rTMS & Pre-rTMS & Post-rTMS & Pre-rTMS & Post-rTMS \\
\hline \multicolumn{7}{|l|}{ Brunnstrom stage } \\
\hline Proximal upper extremity (1-6) & $3.40 \pm 1.47$ & $3.60 \pm 1.31^{*}$ & $3.38 \pm 1.26$ & $3.38 \pm 1.26$ & $3.13 \pm 1.41$ & $3.13 \pm 1.41$ \\
\hline Distal upper extremity (1-6) & $3.15 \pm 1.60$ & $3.25 \pm 1.48$ & $3.25 \pm 1.48$ & $3.25 \pm 1.48$ & $2.93 \pm 1.53$ & $2.93 \pm 1.53$ \\
\hline \multicolumn{7}{|l|}{ MRC } \\
\hline Shoulder flexion (0-5) & $3.13 \pm 1.18$ & $3.70 \pm 1.21^{* * *, \dagger}$ & $2.88 \pm 1.48$ & $3.28 \pm 1.34^{* *}$ & $2.70 \pm 1.41$ & $2.83 \pm 1.56$ \\
\hline Elbow extension $(0-5)$ & $2.43 \pm 1.22$ & $2.85 \pm 1.37^{* *} \dagger^{\dagger}$ & $2.75 \pm 1.52$ & $2.91 \pm 1.53$ & $2.37 \pm 1.49$ & $2.43 \pm 1.59$ \\
\hline Elbow flexion $(0-5)$ & $3.33 \pm 1.14$ & $3.85 \pm 0.99^{* * *, \dagger}$ & $3.34 \pm 1.29$ & $3.72 \pm 1.28^{* *}$ & $2.70 \pm 1.41$ & $2.83 \pm 1.50$ \\
\hline Wrist extension $(0-5)$ & $1.15 \pm 1.01$ & $1.28 \pm 1.13^{*}$ & $1.53 \pm 1.31$ & $1.66 \pm 1.45$ & $1.47 \pm 1.30$ & $1.47 \pm 1.30$ \\
\hline Wrist flexion $(0-5)$ & $1.15 \pm 1.11$ & $1.28 \pm 1.24^{*}$ & $1.56 \pm 1.41$ & $1.63 \pm 1.46$ & $1.47 \pm 1.30$ & $1.50 \pm 1.30$ \\
\hline Grasp $(0-5)$ & $1.78 \pm 1.14$ & $2.48 \pm 1.14^{* * * \dagger}$ & $2.41 \pm 1.26$ & $2.97 \pm 1.26^{* * *, \#}$ & $1.87 \pm 1.41$ & $2.03 \pm 1.48$ \\
\hline \multicolumn{7}{|l|}{ FMA } \\
\hline Total $(0-126)$ & $82.50 \pm 18.82$ & $85.95 \pm 17.85^{* * * \dagger}$ & $86.5 \pm 18.68$ & $90.69 \pm 18.01^{* *, \#}$ & $85.67 \pm 21.85$ & $86.00 \pm 21.95^{*}$ \\
\hline Upper extremity $(0-36)$ & $19.80 \pm 8.89$ & $21.90 \pm 8.06^{* * *}$ & $19.56 \pm 11.08$ & $22.06 \pm 10.66^{*, \#}$ & $18.33 \pm 11.94$ & $18.47 \pm 11.99$ \\
\hline Wrist $(0-10)$ & $1.15 \pm 2.43$ & $1.25 \pm 2.48$ & $2.44 \pm 3.20$ & $2.56 \pm 3.27$ & $2.47 \pm 2.97$ & $2.47 \pm 2.97$ \\
\hline Hand $(0-14)$ & $3.10 \pm 4.61$ & $3.35 \pm 4.48^{*}$ & $5.25 \pm 4.93$ & $5.88 \pm 5.12^{* *, \#}$ & $5.00 \pm 5.18$ & $5.13 \pm 5.29$ \\
\hline Coordination and speed (0-6) & $2.20 \pm 1.82$ & $2.50 \pm 2.09$ & $2.25 \pm 1.95$ & $2.44 \pm 1.86$ & $2.40 \pm 1.96$ & $2.13 \pm 2.00$ \\
\hline Sensation $(0-12)$ & $10.90 \pm 2.94$ & $11.30 \pm 1.72$ & $11.69 \pm 1.01$ & $11.75 \pm 1.00$ & $11.87 \pm 0.52$ & $11.87 \pm 0.52$ \\
\hline Passive joint motion (0-24) & $23.00 \pm 1.49$ & $23.10 \pm 1.29$ & $23.25 \pm 1.29$ & $23.63 \pm 1.09$ & $23.20 \pm 2.60$ & $23.20 \pm 2.60$ \\
\hline Joint pain $(0-24)$ & $22.35 \pm 1.98$ & $22.80 \pm 1.54$ & $22.06 \pm 3.02$ & $22.38 \pm 2.99$ & $22.40 \pm 3.70$ & $22.40 \pm 3.70$ \\
\hline \multicolumn{7}{|l|}{ WMFT } \\
\hline Total $(0-75)$ & $24.50 \pm 20.99$ & $29.10 \pm 21.96^{* *, \dagger}$ & $27.63 \pm 20.63$ & $30.88 \pm 21.97^{* *, * \#}$ & $28.67 \pm 25.34$ & $29.20 \pm 25.43$ \\
\hline Forearm to table $(0-5)$ & $2.65 \pm 1.50$ & $3.20 \pm 1.40^{* * *}$ & $2.75 \pm 1.44$ & $3.25 \pm 1.34^{* *}$ & $2.67 \pm 1.50$ & $2.93 \pm 1.67^{*}$ \\
\hline Forearm to box $(0-5)$ & $2.35 \pm 1.81$ & $2.85 \pm 1.81^{* * *}$ & $2.31 \pm 1.53$ & $2.56 \pm 1.67$ & $2.40 \pm 1.77$ & $2.53 \pm 1.89$ \\
\hline Extend elbow $(0-5)$ & $1.90 \pm 1.53$ & $2.50 \pm 1.57^{* * *, \dagger,+}$ & $2.25 \pm 1.84$ & $2.31 \pm 1.85$ & $2.27 \pm 1.79$ & $2.20 \pm 1.74$ \\
\hline Extend elbow (weight) (0-5) & $1.65 \pm 1.57$ & $2.10 \pm 1.86^{*, \dagger,+}$ & $2.19 \pm 1.80$ & $2.19 \pm 1.80$ & $2.07 \pm 1.91$ & $2.07 \pm 1.87$ \\
\hline Hand to table $(0-5)$ & $2.70 \pm 1.46$ & $3.10 \pm 1.65^{* *}$ & $2.63 \pm 1.46$ & $3.00 \pm 1.76$ & $2.47 \pm 1.69$ & $2.60 \pm 1.77$ \\
\hline Hand to box $(0-5)$ & $2.25 \pm 1.80$ & $2.60 \pm 1.93^{* *}$ & $2.19 \pm 1.68$ & $2.56 \pm 1.79^{\#}$ & $2.20 \pm 1.934$ & $2.20 \pm 1.934$ \\
\hline Weight to box & $1.95 \pm 1.67$ & $2.25 \pm 1.92^{* *, \dagger}$ & $1.88 \pm 1.75$ & $2.00 \pm 1.79$ & $2.00 \pm 1.85$ & $2.00 \pm 1.85$ \\
\hline Reach and retrieve $(0-5)$ & $3.15 \pm 1.14$ & $3.90 \pm 0.97^{* * *, \dagger}$ & $3.19 \pm 1.28$ & $3.75 \pm 1.29^{* * * \#}$ & $2.87 \pm 1.77$ & $3.00 \pm 1.85$ \\
\hline Lift can $(0-5)$ & $1.20 \pm 1.58$ & $1.35 \pm 1.53$ & $1.56 \pm 1.71$ & $1.69 \pm 1.81$ & $1.60 \pm 1.96$ & $1.80 \pm 2.08$ \\
\hline Lift pencil $(0-5)$ & $0.70 \pm 1.30$ & $0.80 \pm 1.44$ & $1.06 \pm 1.29$ & $1.19 \pm 1.38$ & $1.13 \pm 1.51$ & $1.13 \pm 1.51$ \\
\hline Lift paper clip $(0-5)$ & $0.70 \pm 1.17$ & $0.85 \pm 1.35$ & $0.88 \pm 1.03$ & $1.19 \pm 1.47$ & $1.07 \pm 1.44$ & $1.00 \pm 1.36$ \\
\hline Stack checkers $(0-5)$ & $0.70 \pm 1.34$ & $0.75 \pm 1.37$ & $0.75 \pm 0.93$ & $0.94 \pm 1.24$ & $1.00 \pm 1.41$ & $1.00 \pm 1.41$ \\
\hline Flip cards $(0-5)$ & $0.45 \pm 0.95$ & $0.55 \pm 1.19$ & $0.63 \pm 0.89$ & $0.75 \pm 1.00$ & $0.87 \pm 1.30$ & $0.80 \pm 1.27$ \\
\hline Turn key in lock $(0-5)$ & $0.65 \pm 1.39$ & $0.65 \pm 1.39$ & $1.00 \pm 1.27$ & $1.06 \pm 1.34$ & $1.13 \pm 1.60$ & $1.07 \pm 1.62$ \\
\hline
\end{tabular}


Table 3 continued

\begin{tabular}{|c|c|c|c|c|c|c|}
\hline & \multicolumn{2}{|c|}{ fNIRS $(n=20)$} & \multicolumn{2}{|l|}{$\operatorname{MEP}(n=16)$} & \multicolumn{2}{|c|}{ Sham $(n=15)$} \\
\hline & Pre-rTMS & Post-rTMS & Pre-rTMS & Post-rTMS & Pre-rTMS & Post-rTMS \\
\hline Fold towel $(0-5)$ & $0.70 \pm 1.42$ & $0.80 \pm 1.51$ & $1.13 \pm 1.46$ & $1.31 \pm 1.66$ & $1.47 \pm 1.69$ & $1.40 \pm 1.68$ \\
\hline Lift basket $(0-5)$ & $0.75 \pm 1.52$ & $0.80 \pm 1.58$ & $1.25 \pm 1.57$ & $1.38 \pm 1.75$ & $1.47 \pm 1.89$ & $1.47 \pm 1.89$ \\
\hline Grip strength $(\mathrm{kg})$ & $5.35 \pm 4.18$ & $6.71 \pm 5.36^{* * \dagger} \dagger$ & $5.28 \pm 5.23$ & $6.34 \pm 5.98^{* *}$ & $3.88 \pm 3.81$ & $3.96 \pm 3.88$ \\
\hline
\end{tabular}

Intragroup comparison was made using a paired $t$ test; intergroup comparison was made using one-way ANOVA and a post hoc analysis with Bonferroni's correction

$U E$ upper extremity, MRC Medical Research Council scale, FMA Fugl-Meyer Assessment, WMFT Wolf Motor Function Test Significance level: intragroup improvement ${ }^{* * *} P<0.001 ;{ }^{* *} P<0.01 ;{ }^{*} P<0.05$

Intergroup comparison: ANIRS superior to sham $\left({ }^{\dagger} P<0.05\right)$, ANIRS superior to MEP $\left({ }^{\star} P<0.05\right)$, MEP superior to sham $\left({ }^{\#} P<0.05\right)$

${ }^{*} P<0.05$, Spearman's correlation

imbalance exists in most patients with chronic stroke [36, 37], rTMS modulates cortical excitability and harnesses neuroplasticity under the operative mechanism of LTP or LTD. Regardless of the contralesional or ipsilesional approach, determination of RMT in the hotspot of the M1 area is key for accessing cortical excitability, and stimulation intensity is defined by RMT accordingly. However, the elicited MEP in hand muscles is usually absent in most cases, partly because of cortical necrosis and partly because of a high motor evoked threshold exceeding the maximal machine output. In such cases, the researchers set the mirror part of the contralesional hotspot as the treatment target, despite there being topographical asymmetry for bilateral M1. Furthermore, in some patients with vascular encephalopathy or elderly patients, the MEP could not be measurable in the unaffected cortex. Therefore, a surrogate for traditional MEP navigation is critical for optimizing rTMS treatment. As a reliable measure of regional cortical hemodynamic change, fNIRS has been widely used in the field of neuroscience or neurorehabilitation to investigate timely hemodynamic change following an active behavior task or passive transcranial magnetic or electric stimulation. Over the last three decades, numerous studies have been conducted on NIRS in a functional neuroimaging approach in several medical fields, including stroke [14], dementia [38], Alzheimer's disease [39], Parkinson's disease [40], psychiatric diseases, and cognition [41]. The hybrid EEG-fNIRS brain computer interface has also enhanced the classification accuracy of hemodynamic change in neural source detection $[20,42]$. The present study is the first to explore the efficacy of this tool as a topographical navigator for target localization prior to high-frequency rTMS treatment in the ipsilesional hemisphere of patients with stroke.

\section{fNIRS-Guided Hotspot Exists in Each Patient}

In this study, fNIRS-HS was observed in each patient, even though in some cases MEP was absent in the hand muscle. This implies that in patients with cortical or subcortical lesions, where MEP-HS is unobtainable, we can locate the most vigorous $\mathrm{HbO}$ change with the fNIRS method. fNIRS measures cortical oxygenation status to neural activity, whereas MEP-HS measures the loci with the strongest functional control through the corticospinal pathway to the terminal muscles [35]. The MEP from hand recording could be hampered by impaired cervical tract continuity or sedative medication consumption rather than a genu cortical lesion. From this viewpoint, fNIRS-HS may be more accessible than MEP-HS.

Patients with ischemic stroke have higher $\mathrm{HbO}$ and HbR levels in the recanalized or nonrecanalized region compared with the neighboring nonischemic area [43]. In the case of recanalization, a preferential distribution of flow to the ischemic area where vascular resistance is lower would explain the higher concentration of $\mathrm{HbO}$ and $\mathrm{HbR}$ compared with the 


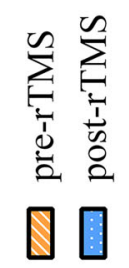
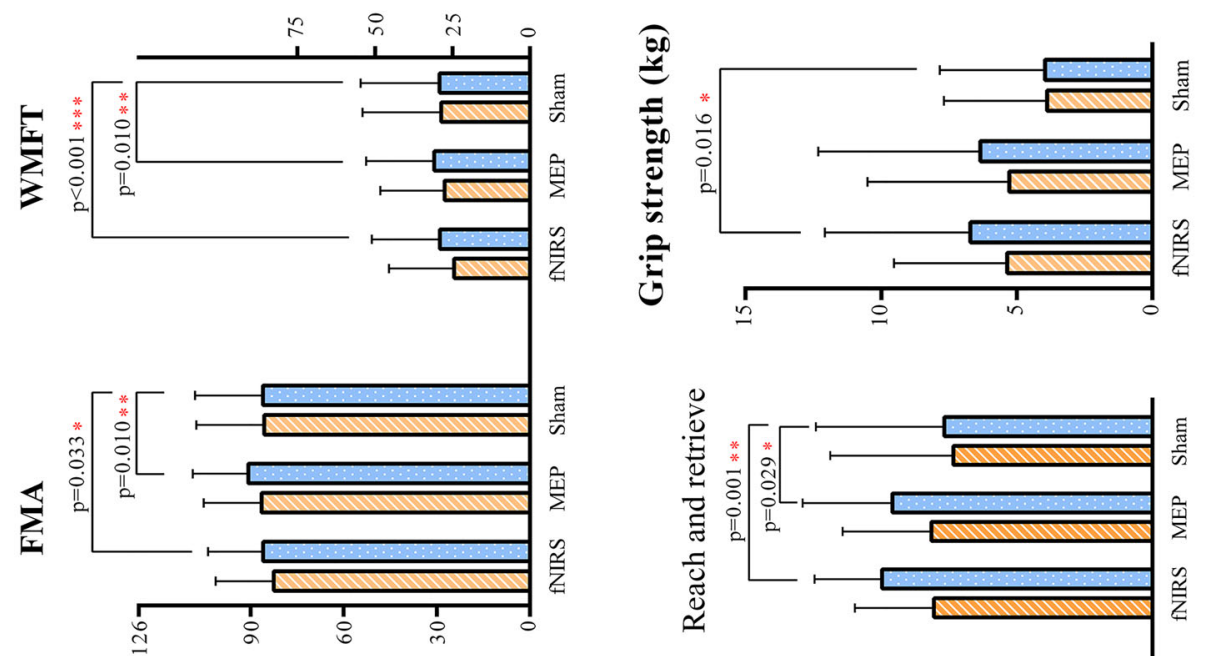

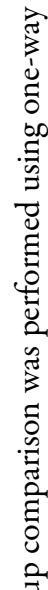
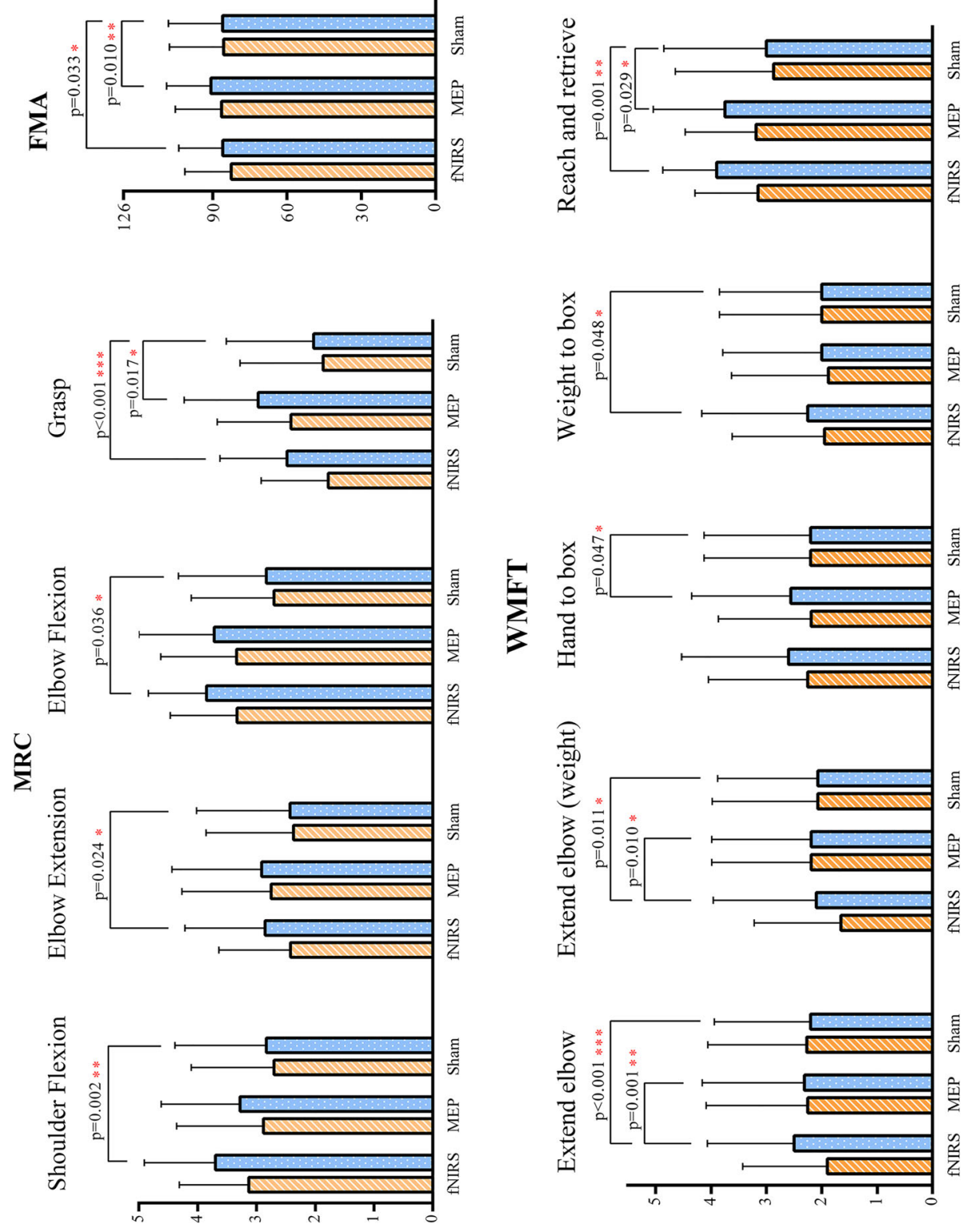

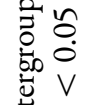

$\stackrel{\breve{\Xi}}{\Xi}$

Е

范 V

战

节

ㄴ

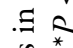

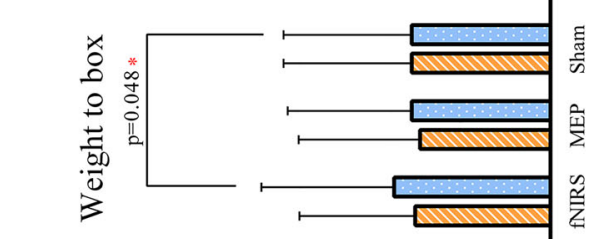

䑰

离

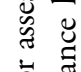

홍

댕

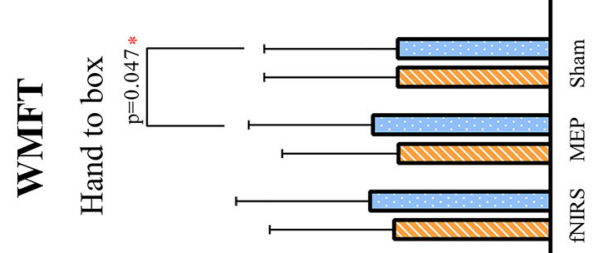

气.

ปิ

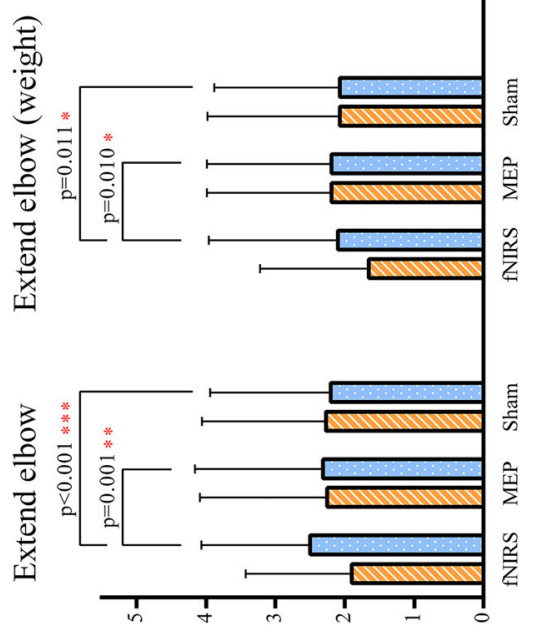

跑

들

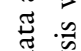

올

ప็ ฮ

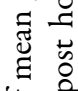

पै

볼

艺

的方 
neighboring nonischemic area. In cases of nonrecanalization, $\mathrm{HbO}$ remains detectable because of residual blood flow from leptomeningeal arterial and venous collaterals, although reduced metabolism and oxygen consumption are noted. Noguchi et al. suggested a significant $\mathrm{HbO}$ increase after single-pulse TMS applied at M1 even though no MEP was detectable [44]. Consistent with these prior findings, we observed that $\mathrm{HbO}$ increased with the rising peak in each patient who had heterogeneous cortical or subcortical lesions. We demonstrated that fNIRS is a feasible tool for the detection of active cortical loci or a hub undergoing reorganization and demanding timely oxygen supply during motor activity.

\section{Distance Between fNIRS-Guided and MEP- Guided Hotspots}

In our study, we observed fNIRS-HS to be very close to MEP-HS. The mean distance between these targets is $2.5 \mathrm{~mm}$ on the $X$ axis and $3 \mathrm{~mm}$ on the $Y$ axis. fNIRS-HS sat more medially and posteriorly than MEP-HS. The reason for this trend could be related to the synergistic pattern from elbow co-activation when the patients were asked to clench their hands during fNIRS assessment. Most of our patients were in Brunnstrom stages II-IV in proximal $(72.5 \%)$ and distal (62.7\%) upper limbs, in whom a synergistic pattern was observed. In a cortical homunculus map representing body part control, M1 for elbow control lies medial and posterior, related to the M1 for hand control. A possible alternative explanation for the posterior shifting of the fNIRS-HS relative to the MEPHS is the activated channels covering the postcentral area (i.e., the somatosensory gyrus in addition to M1) during voluntary hand movement in healthy participants [31]. This is similar to the finding of Herwig et al., who reported that the voluntary finger movement during fMRI involved activation in the somatosensory cortex, which may account for the posterior location of the fNIRS-HS relative to MEP-HS [45]. Despite the spatial discrepancy between the fNIRS-HS and MEP-HS, we observed that a closer distance to the mean MEP-HS was associated with greater improvement in elbow extensor strength. This result indicates that rTMS treatment assisted by fNIRS mapping is a reliable method. fNIRS-HS can serve as a surrogate for standard MEP-HS, particularly for patients in whom the MEP-HS is undetectable.

\section{$\Delta \mathrm{HbO}$ Correlates Inversely with RMT}

We observed that some of the fNIRS channels exhibited significant correlations with RMT, which was distributed in the M1 and S1 regions. Our findings agree with previous results showing that $\mathrm{Hb}$ concentration changes were more prominent at M1 and areas posterior to it [18]. Another study investigating $\mathrm{HbO}$ change during fNIRS recording in healthy participants indicated that the channels with a significant increase in $\mathrm{HbO}$ during finger-tapping tasks mainly covered the primary motor, somatosensory cortex, and supplementary motor areas [29]. In our study, task-related coactivation as reflected in increased $\mathrm{HbO}$ was noted in surrounding M1 areas, including S1 and the prefrontal cortex. The coactivation in these regions may be caused by the interconnected pathway between the primary motor and sensory cortex and the prefrontal cortex [46]. The neurovascular coupling could be influenced by corticocortical and subcorticocortical connections between the motor and somatosensory cortices [18]. Among the 23 channels in our fNIRS montage, four exhibited a significant correlation with the increase in $\mathrm{HbO}$ and the RMT values, with three lying in M1 and one in S1. The intimate relationship between $\mathrm{HbO}$ and RMT indicates the high specificity of fNIRS for use as an RMT surrogate. We assume that the channel with the most vigorous $\mathrm{HbO}$ increase in the M1 territory represents the optimal loci for efficient modulation-rTMS treatment.

\section{fNIRS Navigation Achieved a Better Outcome in Elbow Strength than the MEP Method}

After iTBS was applied to the ipsilesional hotspots, both intervention groups exhibited 
significant a improvement in muscle strength assessed using MRC or grip dynamometer. The fNIRS group exhibited particularly improved elbow strength compared with the MEP group. Furthermore, both intervention groups achieved proximal and hand improvement in FMA and WMFT total scores and sub-tests, with the fNIRS method preferentially enhancing the elbow extension function compared with the MEP method. As mentioned, at the group level, fNIRS-HS tends to have a posteromedial deviation compared with the location of MEP-HS. The deviation pointing to the M1 controlling elbow movement in the cortical homunculus map may be the reason for this superiority. Empirically, the weakness of the elbow extensor complicated by biceps spasticity is the nidus that causes stagnation for post-stroke motor recovery. The functional restoration in the elbow extension is a key step for synergistic pattern breaking and subsequent progression to the next recovery stage.

Notably, although the fNIRS group achieved superior outcome in the elbow, this method improved hand function in magnitude comparable to that in the MEP group. With the experience from this study, in the future we may investigate the effects of fNIRS navigation to examine the influence if we instruct patients to co-contract the elbow muscles as little as possible.

In this study, we did not observe any alteration in scores assessing fine motor control in WMFT in any group, such as lifting a pencil, flipping cards, or turning a key in a lock. Because fine motor control is mediated by complex interregional connection and integration of secondary cortical motor or subcortical circuits, the dexterity function enhanced by the rTMS protocol may need more conditioning sessions to be addressed on these measuring scales. In our experience, the improvement in hand dexterity can be shown after the twocourse rTMS protocol.

This study had some limitations. First, our sample size, albeit the largest for combined NIRS and rTMS studies in stroke so far, was limited. Second, the patients had different lesion types, locations, and post-stroke durations, making it challenging to precisely determine the effect of fNIRS-guided rTMS treatment in specific subgroups. Further largescale studies involving patients of specific stroke types are warranted. Third, compared with a previous study in which double-dense and quadruple-dense fNIRS were used to enhance spatial resolution at the centimeter level [47], we used a single standard density array, which may have increased spatial bias for fNIRS-HS detection. To improve the reliability of this novel guidance method, a double-dense array setting should be ideal. In addition, the effects of one-course rTMS conditioning may last for several months [7]. Fourth, we did not follow up with patients to investigate the enduring effect. Future studies should include a long-term follow-up to determine the overall efficacy of the fNIRS-aided system.

\section{CONCLUSIONS}

In this RCT, we successfully explored the feasibility of the fNIRS guidance system applied in patients with stroke for motor neuromodulation. This system has the advantages of being relatively cost-effective, portable, and reliable in navigation, with the possibility of digitalized software integration; therefore, it may serve as a surrogate biomarker for cortical excitability. This novel method achieved better functional restoration in the proximal upper limb than the MEP method. The incorporation of machine learning and automatic software analysis into the fNIRS system could facilitate precision medicine for individualized planning before rTMS treatment.

\section{ACKNOWLEDGEMENTS}

We thank all the participants of the study.

Funding. This study and the journal's Rapid Service Fee were supported by Ministry of Science and Technology of Taiwan (108-2314-B075-047, 107-2314-B-075-010) and Taipei Veterans General Hospital (V105C-042). 
Medical Writing and Editorial Assistance. Sudeep Agarwal and Jane Nicholson (Wallace Academic Editing, Taipei, Taiwan) provided medical writing editorial support. This assistance was funded by Taipei Veterans General Hospital (Taipei, Taiwan).

Authorship. All named authors meet the International Committee of Medical Journal Editors (ICMJE) criteria for authorship for this article, take responsibility for the integrity of the work as a whole, and have given their approval for this version to be published.

Authors' Contributions. Po-Yi Tsai, ChiaFeng Lu, and Shin-Tsu Chang contributed to the study conception and design and to the literature search. The analysis and interpretation of results was performed by Pang-Wei Chang, Chia-Feng Lu, and Po-Yi Tsai. The first draft of the manuscript was written by Pang-Wei Chang and Po-Yi Tsai, and all authors commented on previous versions of the manuscript. The final draft of the manuscript was written by all authors. All authors have read and agreed to the published version of the manuscript.

Disclosures. Po-Yi Tsai received funds from Ministry of Science and Technology of Taiwan (108-2314-B-075-047, 107-2314-B-075-010) and Taipei Veterans General Hospital (V105C-042). Pang-Wei Chang, Chia-Feng Lu, and Shin-Tsu Chang have nothing to disclose.

Compliance with Ethics Guidelines. All procedures performed in this study involving human participants were in accordance with the ethical standards of the national research committee and with the 1964 Helsinki Declaration and its later amendments. This study has been approved by the Institutional Review Board of Taipei Veterans General Hospital (201307011A; Registration-URL: https://clinical trials.gov; Unique identifier: NCT02006615). Informed consent was obtained from all individual participants included in the study.

Data Availability. The datasets generated during and/or analyzed during the current study are available from the corresponding author on reasonable request.

Open Access. This article is licensed under a Creative Commons Attribution-NonCommercial 4.0 International License, which permits any non-commercial use, sharing, adaptation, distribution and reproduction in any medium or format, as long as you give appropriate credit to the original author(s) and the source, provide a link to the Creative Commons licence, and indicate if changes were made. The images or other third party material in this article are included in the article's Creative Commons licence, unless indicated otherwise in a credit line to the material. If material is not included in the article's Creative Commons licence and your intended use is not permitted by statutory regulation or exceeds the permitted use, you will need to obtain permission directly from the copyright holder. To view a copy of this licence, visit http://creativecommons.org/licenses/by$\mathrm{nc} / 4.0 /$.

\section{REFERENCES}

1. Virani SS, Alonso A, Benjamin EJ, et al. Heart disease and stroke statistics-2020 update: a report from the American Heart Association. Circulation. 2020;141(9):e139-596.

2. Hoogendam JM, Ramakers GM, Di Lazzaro V. Physiology of repetitive transcranial magnetic stimulation of the human brain. Brain Stimul. 2010;3(2):95-118.

3. Hsu WY, Cheng CH, Liao KK, Lee IH, Lin YY. Effects of repetitive transcranial magnetic stimulation on motor functions in patients with stroke: a metaanalysis. Stroke. 2012;43(7):1849-57.

4. Huang YZ, Edwards MJ, Rounis E, Bhatia KP, Rothwell JC. Theta burst stimulation of the human motor cortex. Neuron. 2005;45(2):201-6.

5. Iezzi E, Suppa A, Conte A, Li Voti P, Bologna M, Berardelli A. Short-term and long-term plasticity interaction in human primary motor cortex. Eur J Neurosci. 2011;33(10):1908-15.

6. Ackerley SJ, Stinear CM, Barber PA, Byblow WD. Combining theta burst stimulation with training after subcortical stroke. Stroke. 2010;41(7):1568-72. 
7. Dionísio A, Duarte IC, Patrício M, Castelo-Branco $\mathrm{M}$. The use of repetitive transcranial magnetic stimulation for stroke rehabilitation: a systematic review. J Stroke Cerebrovasc Dis. 2018;27(1):1-31.

8. Lefaucheur JP, Aleman A, Baeken C, et al. Evidencebased guidelines on the therapeutic use of repetitive transcranial magnetic stimulation (rTMS): An update (2014-2018). Clin Neurophysiol. 2020;131(2):474-528.

9. Hamada M, Murase N, Hasan A, Balaratnam M, Rothwell JC. The role of interneuron networks in driving human motor cortical plasticity. Cereb Cortex. 2013;23(7):1593-605.

10. Nettekoven C, Volz LJ, Leimbach M, et al. Interindividual variability in cortical excitability and motor network connectivity following multiple blocks of rTMS. Neuroimage. 2015;118:209-18.

11. Huo C, Xu G, Li Z, et al. Limb linkage rehabilitation training-related changes in cortical activation and effective connectivity after stroke: a functional near-infrared spectroscopy study. Sci Rep. 2019;9(1):6226.

12. Ferrari M, Quaresima V. A brief review on the history of human functional near-infrared spectroscopy (fNIRS) development and fields of application. Neuroimage. 2012;63(2):921-35.

13. Villringer A, Chance B. Non-invasive optical spectroscopy and imaging of human brain function. Trends Neurosci. 1997;20(10):435-42.

14. Yang M, Yang Z, Yuan T, Feng W, Wang P. A systemic review of functional near-infrared spectroscopy for stroke: current application and future directions. Front Neurol. 2019;10:58.

15. Drenckhahn C, Koch SP, Dümmler J, Kohl-Bareis M, Steinbrink J, Dreier JP. A validation study of the use of near-infrared spectroscopy imaging in primary and secondary motor areas of the human brain. Epilepsy Behav. 2015;49:118-25.

16. Koenraadt KL, Munneke MA, Duysens J, Keijsers NL. TMS: a navigator for NIRS of the primary motor cortex? J Neurosci Methods. 2011;201(1):142-8.

17. Curtin A, Tong S, Sun J, Wang J, Onaral B, Ayaz H. A systematic review of integrated functional near-infrared spectroscopy (fNIRS) and transcranial magnetic stimulation (TMS) studies. Front Neurosci. 2019;13:84.

18. Furubayashi T, Mochizuki H, Terao Y, et al. Cortical hemoglobin concentration changes underneath the coil after single-pulse transcranial magnetic stimulation: a near-infrared spectroscopy study. J Neurophysiol. 2013;109(6):1626-37.
19. Quaresima V, Ferrari M. Functional near-infrared spectroscopy (fNIRS) for assessing cerebral cortex function during human behavior in natural/social situations: a concise review. Organ Res Methods. 2019;22(1):46-68.

20. Cao J, Huppert TJ, Grover P, Kainerstorfer JM. Enhanced spatiotemporal resolution imaging of neuronal activity using joint electroencephalography and diffuse optical tomography. Neurophotonics. 2021;8(1): 015002 .

21. Sharma G, Roy CS. Design of NIRS probe based on computational model to find out the optimal location for non-invasive brain stimulation. J Med Syst. 2018;42(12):244.

22. Faul F, Erdfelder E, Lang AG, Buchner A. G*Power 3: a flexible statistical power analysis program for the social, behavioral, and biomedical sciences. Behav Res Methods. 2007;39(2):175-91.

23. Schmitz CH, Klemer DP, Hardin R, et al. Design and implementation of dynamic near-infrared optical tomographic imaging instrumentation for simultaneous dual-breast measurements. Appl Opt. 2005;44(11):2140-53.

24. Kocsis L, Herman P, Eke A. The modified Beer-Lambert law revisited. Phys Med Biol. 2006;51(5):N91-8.

25. Piper SK, Krueger A, Koch SP, et al. A wearable multi-channel fNIRS system for brain imaging in freely moving subjects. Neuroimage. 2014;85 Pt 1(1):64-71.

26. Antoniadis A, Bigot J, Sapatinas T. Wavelet estimators in nonparametric regression: a comparative simulation study. J Stat Softw. 2001;6(6):1-83.

27. Chipman HA, Kolaczyk ED, McCulloch RE. Adaptive bayesian wavelet shrinkage. J Am Stat Assoc. 1997;92(440):1413-21.

28. Molavi B, Dumont GA. Wavelet-based motion artifact removal for functional near-infrared spectroscopy. Physiol Meas. 2012;33(2):259-70.

29. Cui X, Bray S, Reiss AL. Functional near infrared spectroscopy (NIRS) signal improvement based on negative correlation between oxygenated and deoxygenated hemoglobin dynamics. Neuroimage. 2010;49(4):3039-46.

30. Huppert TJ, Diamond SG, Franceschini MA, Boas DA. HomER: a review of time-series analysis methods for near-infrared spectroscopy of the brain. Appl Opt. 2009;48(10):D280-98.

31. Jiang Y, Li Z, Zhao Y, et al. Targeting brain functions from the scalp: Transcranial brain atlas based 
on large-scale fMRI data synthesis. Neuroimage. 2020;210: 116550 .

32. Fugl-Meyer AR, Jääskö L, Leyman I, Olsson S, Steglind $\mathrm{S}$. The post-stroke hemiplegic patient. 1. A method for evaluation of physical performance. Scand J Rehabil Med. 1975;7(1):13-31.

33. Wolf SL, Catlin PA, Ellis M, Archer AL, Morgan B, Piacentino A. Assessing Wolf motor function test as outcome measure for research in patients after stroke. Stroke. 2001;32(7):1635-9.

34. Wolf SL, Thompson PA, Morris DM, et al. The EXCITE trial: attributes of the Wolf Motor Function Test in patients with subacute stroke. Neurorehabil Neural Repair. 2005;19(3):194-205.

35. Rossini PM, Burke D, Chen R, et al. Non-invasive electrical and magnetic stimulation of the brain, spinal cord, roots and peripheral nerves: Basic principles and procedures for routine clinical and research application. An updated report from an I.F.C.N. Committee. Clin Neurophysiol. 2015;126(6):1071-107.

36. Hummel FC, Celnik P, Pascual-Leone A, Fregni F, Byblow WD, Buetefisch CM, et al. Controversy: noninvasive and invasive cortical stimulation show efficacy in treating stroke patients. Brain Stimul. 2008;1:370-82.

37. Murase N, Duque J, Mazzocchio R, Cohen LG. Influence of interhemispheric interactions on motor function in chronic stroke. Ann Neurol. 2004;55:400-9.

38. Yeung MK, Chan AS. Functional near-infrared spectroscopy reveals decreased resting oxygenation levels and task-related oxygenation changes in mild cognitive impairment and dementia: a systematic review. J Psychiatr Res. 2020;124:58-76.

39. Fallgatter AJ, Roesler M, Sitzmann L, Heidrich A, Mueller TJ, Strik WK. Loss of functional hemispheric asymmetry in Alzheimer's dementia assessed with near-infrared spectroscopy. Brain Res Cogn Brain Res. 1997;6(1):67-72.

40. Stuart S, Vitorio R, Morris R, Martini DN, Fino PC, Mancini M. Cortical activity during walking and balance tasks in older adults and in people with Parkinson's disease: a structured review. Maturitas. 2018;113:53-72.

41. Pinti P, Tachtsidis I, Hamilton A, et al. The present and future use of functional near-infrared spectroscopy (fNIRS) for cognitive neuroscience. Ann N Y Acad Sci. 2020;1464(1):5-29.

42. Fazli S, Mehnert J, Steinbrink J, et al. Enhanced performance by a hybrid NIRS-EEG brain computer interface. Neuroimage. 2012;59(1):519-29.

43. Giacalone G, Zanoletti M, Re R, et al. Time-domain near-infrared spectroscopy in acute ischemic stroke patients. Neurophotonics. 2019;6(1): 015003.

44. Noguchi Y, Watanabe E, Sakai KL. An event-related optical topography study of cortical activation induced by single-pulse transcranial magnetic stimulation. Neuroimage. 2003;19(1):156-62.

45. Herwig U, Kölbel K, Wunderlich AP, et al. Spatial congruence of neuronavigated transcranial magnetic stimulation and functional neuroimaging. Clin Neurophysiol. 2002;113(4):462-8.

46. Enomoto H, Ugawa Y, Hanajima R, et al. Decreased sensory cortical excitability after $1 \mathrm{~Hz}$ rTMS over the ipsilateral primary motor cortex. Clin Neurophysiol. 2001;112(11):2154-8.

47. Yamamoto T, Maki A, Kadoya T, et al. Arranging optical fibres for the spatial resolution improvement of topographical images. Phys Med Biol. 2002;47(18):3429-40. 